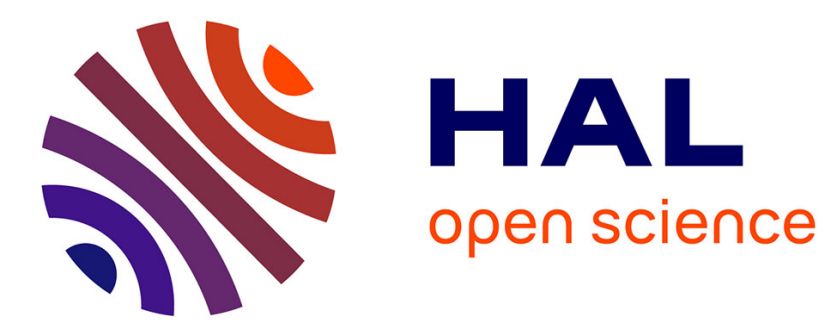

\title{
La pleine conscience au travail : une revue systématique de la littérature
}

Christophe Czuly, Fanny Poujol

\section{To cite this version:}

Christophe Czuly, Fanny Poujol. La pleine conscience au travail : une revue systématique de la littérature. @GRH, 2018, 28 (3),pp.97. 10.3917/grh.183.0097 . hal-03121542

\section{HAL Id: hal-03121542 \\ https://hal.parisnanterre.fr/hal-03121542}

Submitted on 29 Jan 2021

HAL is a multi-disciplinary open access archive for the deposit and dissemination of scientific research documents, whether they are published or not. The documents may come from teaching and research institutions in France or abroad, or from public or private research centers.
L'archive ouverte pluridisciplinaire HAL, est destinée au dépôt et à la diffusion de documents scientifiques de niveau recherche, publiés ou non, émanant des établissements d'enseignement et de recherche français ou étrangers, des laboratoires publics ou privés. 


\section{La pleine conscience au travail : une revue systématique de la littérature}

CZULY Christophe, Université de Montpellier

Doctorant en Sciences de Gestion

Montpellier Recherche en Management

163 Rue Auguste Broussonnet, 34090 Montpellier

Téléphone : 0608865598

czuly.christophe@wanadoo.fr

POUJOL Fanny, CEROS, Université Paris-Nanterre, INSEEC Paris

Professeur des Universités

UFR de Sciences Economiques Gestion Mathématiques Informatique (SEGMI) 200, Avenue de la République, 92001 Nanterre

Téléphone : 0671828859

poujol.fanny@laposte.net 


\section{La pleine conscience au travail : une revue systématique de la littérature}

Résumé

L'objectif de ce papier est de présenter les principales avancées sur la pleine conscience en milieu professionnel ces dix dernières années et ses effets en entreprise. Alors que nous assistons à l'émergence d'une littérature dédiée à la pleine conscience, ce thème souffre encore d'un manque de consensus quant à sa conceptualisation. Après avoir clarifié le concept et à partir d'une revue systématique de littérature et d'une analyse de celle-ci, cet article vise à comprendre les effets de la pleine conscience en entreprise. Le traitement des données automatisé a été effectué avec TROPES et l'extension graphique GEPHI. II en résulte un cadre intégratif synthétisant les connaissances sur le sujet et pose les fondations théoriques pour les recherches futures. Les résultats et leur analyse montrent l'importance de la pleine conscience en entreprise de par ses effets sur les réactions et comportements organisationnels contribuant à la performance des employés. Nous proposons également des pistes d'investigation afin de nourrir la dynamique de ce domaine de recherche.

Mots clés: pleine-conscience, comportements organisationnels, bien-être des employés, performance des employés.

Abstract

The objective of this paper is to present the main advances on mindfulness in the workplace over the past ten years and its effects in companies. While we are witnessing the emergence of a literature dedicated to mindfulness, this theme still suffers from a lack of consensus as to its conceptualization. After having clarified the concept and based on a systematic literature review and analysis, this article aims to understand the effects of mindfulness in companies. The automated data processing was done with TROPES and the graphic extension GEPHI. The result is an integrative framework synthesizing knowledge on the subject and lays the theoretical foundation for future research. The results and their analysis show the importance of mindfulness and its effects on organizational reactions and behaviors that impact employees' performance. We also propose avenues of investigation to feed the dynamics of this field of research.

Keywords: mindfulness, organizational behaviors, employee well-being, employee performance. 
Introduction

Le concept de pleine conscience ou mindfulness est complexe car il regroupe les idées de méditation, d'état, de trait, de processus et de pratique thérapeutique. Le site internet Amazon recense plus de 25000 livres actuellement en vente sur la pleine conscience. Une recherche avec le mot mindfulness dans la base de données $\mathrm{ABI} / \mathrm{Inform}$ propose plus de 3000 publications académiques entre 2000 et 2018. Cet engouement pour le sujet de la pleine conscience est notamment dû aux preuves apportées sur la pratique de la méditation et ses effets sur le bien-être des individus (Walsh et Shapiro, 2006). Les interventions sur la pleine conscience, dites contemplatives, visent à renforcer l'attention intentionnelle sur le moment présent, dans l'acceptation et le non jugement. Elles permettent l'obtention de comportements plus flexibles et adaptés aux situations telles qu'elles se présentent en diminuant les réponses automatisées (Grégoire et al., 2016). L'intérêt des sciences pour la pleine conscience dans les domaines de la psychologie et de la médecine est sans appel, mais il existe peu d'études sur la pleine conscience en milieu professionnel (Dane et Brummel, 2013). Quelques recherches cependant, démontrent l'effet positif de la pleine conscience sur la satisfaction au travail (Hülsheger et al., 2013) ou sur les résultats des employés (Glomb et al., 2012). Si le bien-être en entreprise constitue un levier de performance (Grenier et Martin, 2013), les pressions quant à elles sont constantes et les salariés sont sujets au stress et à l'épuisement professionnel (Abord-de-Chatillon et Bachelard, 2006 ; Gintrac, 2011). La performance est un concept polysémique qui peut s'envisager à plusieurs niveaux et termes. Dans ce travail nous nous intéressons aux effets de la pleine conscience sur les réactions et comportements et organisationnels importants en terme de performance des employés. En adoptant une approche systématique, cet article propose une revue de la littérature de la dernière décennie traitant de la pleine conscience au travail. L'objectif est de mieux définir la pleine conscience en clarifiant les connaissances de : 1) ses fondements et processus sous-jacents ; 2) ses effets et notamment en terme de comportements organisationnels. Ce papier se présente en trois points: la revue de littérature, la méthodologie, les résultats. Enfin une discussion conclura ce travail avec les limites et les voies futures de la recherche.

1 La pleine conscience : origine, définitions, pratique et effets

Notre revue de littérature s'inscrit dans le champ de la psychologie moderne avec la troisième vague des Thérapies Cognitivo-Comportementales qui intègrent les programmes de réduction du stress grâce à la pleine conscience dont le MBSR Mindfulness-BasedStress-Reduction (Hayes et al., 2006). La pleine conscience s'inscrit également dans le 
champ des théories de l'organisation qui «visent à aider les dirigeants ou responsables d'organisations [...] à améliorer l'efficience de leur fonctionnement en la rationalisant » (Desreumaux, les finalités de la discipline, 2015 p.3 section 1).

\subsection{Les origines de la pleine conscience}

Le concept de pleine conscience date de plus de 2500 ans et est principalement issu de la philosophie bouddhiste. La pleine conscience a également des parentés avec différents courants philosophiques de l'ancienne Europe de l'ouest (philosophie grecque, existentialisme, phénoménologie, naturalisme), ou de courants humanistes ou transcendantaux en Amérique (Brown et al. 2007). Le phénomène a ainsi inspiré la littérature autant philosophique que religieuse en traversant les siècles et les cultures. Initié par les écrits du bouddhisme 500 ans avant JC, il est repris par l'indouisme entre le $6^{\text {ème }}$ et le $2^{\text {ème }}$ siècle avant JC (Varenne, 1990). Des similarités apparaissent ensuite avec le taoïsme et la philosophie grecque vers le $4^{\text {ème }}$ siècle avant JC (De Bruyn, 2009 ; Pavie, 2010). Viennent alors le soufisme et l'hébraïsme au $3^{\text {ème }}$ siècle avant JC (Kane, 2013 ; Ludwig, 2006). Enfin, le concept émerge au travers de la méditation chrétienne : mouvement de prière fondé par le moine bénédictin anglais John Main qui n'a cessé de croître depuis 1975 (Freeman, 2007). Selon Shapiro et al. (2008), le phénomène de la pleine conscience est naturel dans l'évolution de l'homme. De vieilles fresques indiennes montrant des personnes en train de méditer remonteraient à plus de 4000 ans (Walsh, 1992 p. 32). A proprement parlé, d'après la littérature scientifique, le mot mindfulness vient du pali : sati/satita et du mot védique : smrti, issus des principales langues dans lesquelles sont rédigés les enseignements de Buddha. Ces mots ont été traduits par Davids fin du XIXème siècle par mindfulness. pleine conscience (Davids et Stede, 1959 p. 725/131). La pleine conscience émerge par l'activation d'un mécanisme métacognitif (Shapiro et al. 2008), c'està-dire aux connaissances qu'un individu a de son propre fonctionnement. Ce mécanisme métacognitif consiste à rediriger son attention sur l'instant présent (Ruedy et Schweitzer, 2010). La pleine conscience est ainsi « une stratégie métacognitive » (Thomas, 2006 p. 86). La pleine conscience émerge à partir du moment où l'individu : 1) arrive à reconnaître quand il n'est plus dans l'état de pleine conscience et 2) quand il redirige son attention sur l'expérience immédiate dans le non-jugement et l'acceptation (Erisman et Roemer, 2012). Une fois que l'attention est redirigée sur l'instant présent, l'état de pleine conscience émerge.

1.2 Les définitions et la pratique de la pleine conscience 
La pleine conscience a commencé à faire son apparition dans les écrits scientifiques dans les années 1980. Jon Kabat-Zinn, Professeur de médecine dans le Massachussetts, lance le programme MBSR : Mindfulness-Based-Stress-Reduction. Le programme est basé sur la réduction du stress par des exercices de pleine conscience selon des pratiques de méditation Bouddhistes. Selon Kabat-Zinn (2003 p. 145) : «An operational working definition of mindfulness is: the awareness that emerges through paying attention on purpose, in the present moment, and nonjudgmentally to the unfolding of experience moment by moment.". Et donc, la mindfulness est " Un état de conscience-awareness qui survient en prêtant une attention, de manière intentionnelle, sur l'expérience du moment présent, instant après instant, et dans le non-jugement ". La conscience-awareness correspond à un état de vigilance accru sur soi et l'environnement, une prise de contact direct avec la réalité (Brown et al. 2007). La pleine conscience correspond d'abord à un état, par définition instable. Quand il existe une propension de l'individu à maintenir son attention sur le moment présent, il s'agit alors du trait de pleine conscience (Brown et Ryan, 2003). Le plus souvent il est question du trait ou disposition de l'individu dans les écrits scientifiques et ce trait est accessible à tous. II est notamment accessible par la pratique de la méditation à la pleine conscience (Grégoire et al., 2016, p. 23). Dans le champ de la psychologie cognitive, Ellen Langer, Professeur à Harvard définit la pleine conscience comme : "un processus consistant en la création de nouvelles catégories et distinctions cognitives " (Langer et Moldoveanu, 2000). La prise en compte de nouveaux repères, par l'attention, crée des interruptions dans les processus automatisés et tend à développer la pleine conscience avec des effets similaires à la méditation (Weick et Putnam, 2006).

II existe de nombreux exercices de méditation à la pleine conscience. Un des exercices, dont la pratique est certainement la plus courante, est fondé sur la respiration consciente. En effet, le concept d'esprit incarné est un concept majeur en pleine conscience dans le sens où le corps et l'esprit sont inextricablement connectés (Brown et al., 2007). L'exercice de la respiration consciente stimule le nerf vague et active le système nerveux parasympathique (système freinateur du corps humain) et permet une meilleure détente. Les conséquences d'un point de vue psychologique, est que lorsque l'attention est portée intensément sur la respiration, l'individu se sent pleinement dans le présent pendant la durée de la respiration (Tan, 2015 p. 3). Apprendre à calmer l'esprit en calmant d'abord le corps permet de renforcer l'insula antérieur droit et le cortex préfrontal (Swierczek et Jousse, 2014). Cette partie du cerveau est en relation avec la conscience émotionnelle menant à un degré plus élevé : l'intelligence émotionnelle. Meilleure est la qualité de l'attention sur le souffle, plus les parties du cerveau impliquées dans l'attention et le contrôle de l'action (Cortex préfrontal) se renforcent (Tan, 2015). La méditation a également pour effet de 
calmer l'anxiété et ainsi libérer l'individu du reflexe rotulien de rejet de nouvelles idées (Brendel et al., 2016). En effet, face aux sentiments de crainte et à l'incertitude, les individus développent des modèles inconscients de pensée et de comportement (Karssiens et al., 2014). Quatre axiomes sont nécessaires pour développer la pleine conscience (Figure 1) : 1) l'intention (l'ouverture sur le champ des possibles), 2) l'attention dans l'instant, 3) l'attitude bienveillante, non jugeante et acceptante (Shapiro et al., 2008). Bishop et al. (2004) proposent un $4^{\text {ème }}$ axe : l'orientation à l'expérience, concept qui rejoint la théorie de Ellen Langer (Pirson et al., 2012).

Figure 1 : Le mécanisme de développement de la pleine conscience adapté de Shapiro et

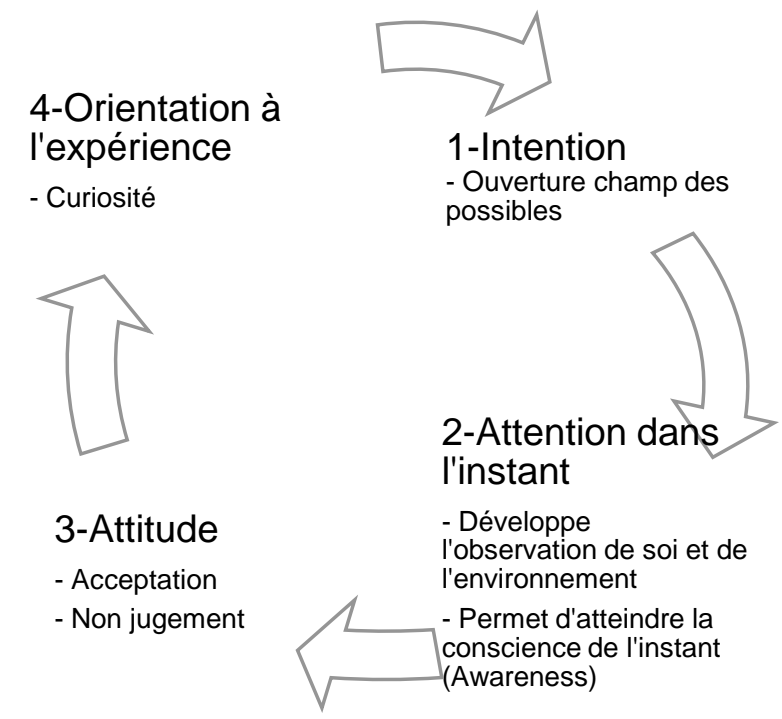

al. (2008) et Bishop et al. (2004)

1.3 Les effets de la pleine conscience sur les comportements organisationnels

De nombreuses études ont démontré que l'entraînement à la pleine conscience a des effets psychologiques, cognitifs et sociaux positifs, sur l'estime de soi, la satisfaction dans la vie, un affect positif, de l'optimisme, un bien être subjectif, une régulation des émotions et des comportements (Akin et al., 2016). La pleine conscience réduit également le stress et l'inquiétude, la réactivité aux émotions, développe la mémoire, la flexibilité cognitive, la compréhension de soi, les relations et les comportements éthiques (Davis et Hayes, 2011) (Figure 2).

Figure 2 : Les effets positifs de la pleine conscience chez l'individu

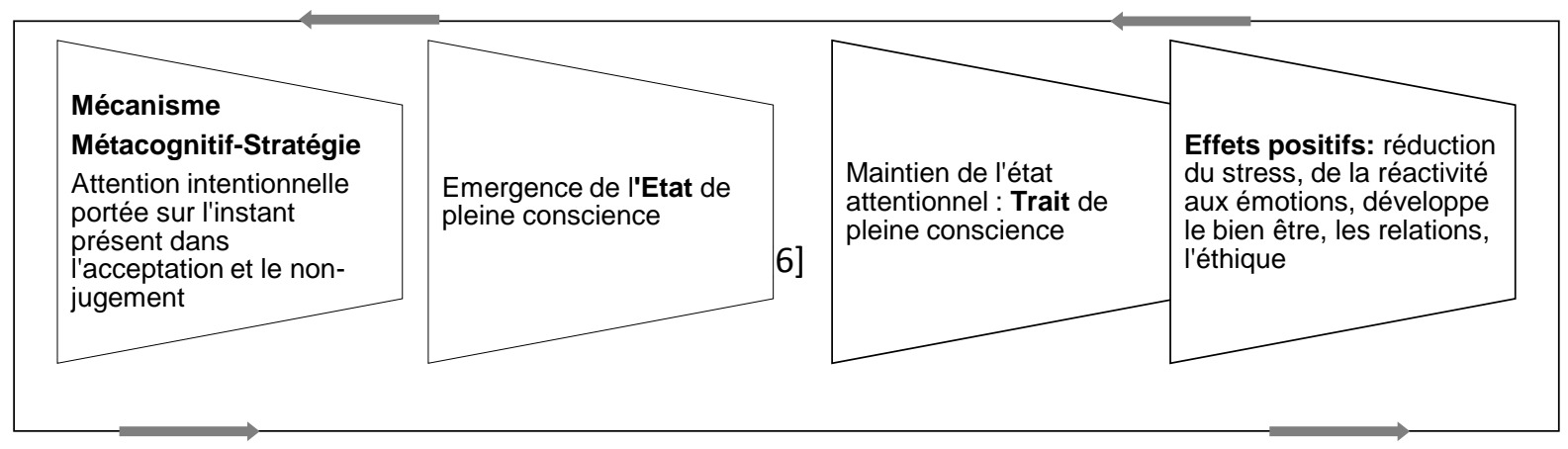


Dans un environnemehric Contrôle de l'attention dans l'instant présent - Pratique majeure des managers, essentielle à la survie de l'organisation. Un certain consensus se dégage de la littérature quant aux comportements organisationnels recherchés pour atteindre les objectifs de l'organisation. Nous pouvons citer ici l'adaptabilité ou les comportements extra rôle des employés. Une autre préoccupation primordiale des managers porte actuellement sur le stress et le bien-être au travail (Abord-de-Chatillon et Bachelard, 2006). La gestion des émotions représente également un enjeu managérial majeur car elles sont en jeu dans le mécanisme du Burnout (Hülsheger et al., 2013 p. 311). Une entreprise qui mise sur le bien-être de ses salariés peut, à la fois développer son capital social et diminuer sa contre-performance (Feuvrier, 2014). Le capital social, étant défini comme « la somme des connaissances et de ressources organisationnelles qui rehaussent la capacité $d u$ potentiel d'action individuelle et collective dans les systèmes sociaux humains " (McElroy et al., 2006). II existe de nombreuses preuves concernant les effets positifs de la pleine conscience sur le bien-être des individus (Walsh et Shapiro, 2006), sur la satisfaction au travail (Hülsheger et al., 2013) et sur différentes catégories de performances : performance de poste (dans le rôle), de tâche, de citoyenneté, de déviance (comportements contre productifs) et de risque d'erreurs (Good et al., 2016 p. 122). Cependant, les recherches sur la pleine conscience en entreprise, si elles se développent, restent encore limitées (Dane, 2011 ; Dane et Brummel, 2013). Les entreprises comme Google, GlaxoSmithKline, Transport-for-London ou PricewaterhouseCoopers reconnaissent ce que la pleine conscience a à offrir aux salariés (Hall, 2013). La pleine conscience peut aider les employés à développer un plus grand bien-être, plus de satisfaction et de meilleures attitudes organisationnelles (Zivnuska et al., 2016). Elle permet un meilleur équilibre du rapport travail famille qui entraîne, à son tour, une plus grande satisfaction au travail du salarié (Allen et Kiburtz, 2012 ; Le Flanchec et al., 2015). Nous pouvons ainsi nous poser la question: Quels sont les effets de la pleine conscience sur les ressources humaines de entreprise?

\section{Le protocole de la revue systématique de la littérature}

La revue systématique de littérature est une méthode qui constitue un élément capital dans les principales avancées des connaissances dans le domaine médical (Durach et al., 2017 p. 67). Elle représente un enjeu pour le développement des connaissances dans d'autres 
sciences comme les sciences de gestion (Tranfield et al., 2003 ; Durach et al., 2017). II s'agit d'une méthode rigoureuse reproductible qui vise à réduire les biais et la partialité dans la sélection de travaux pris en compte. Cette méthode a déjà été mobilisée dans le cadre de recherches sur la pleine conscience au travail mais pour des objectifs différents de cette étude (Françoise, 2016). Notre recherche a un double objectif : 1) procéder à une clarification de l'information concernant les études réalisées sur la pleine conscience en entreprise ces dix dernières années et, 2) connaitre les variables reliées au phénomène. Nous avons procédé en deux temps. Tout d'abord nous avons trié et sélectionné l'information, puis, dans un deuxième temps, nous avons traité et analysé les principales contributions des articles académiques retenus avec l'aide d'un logiciel de traitement de données textuelles.

\subsection{La recherche et l'éligibilité des articles académiques}

Nous nous sommes focalisés sur la littérature scientifique publiée et évaluée par les pairs. Les articles ont d'abord été identifiés à partir des mots clés préalablement définis sur les bases de données bibliographiques suivantes : $\mathrm{ABI}$ inform, Ebsco, Google Scholar, ScienceDirect. Dans un deuxième temps, chaque document présélectionné a été classé dans un dossier. Ensuite les documents ont été sélectionnés et inclus dans une collection bibliographique réalisée avec Excel contenant les références, les résumés et les principaux résultats. La sélection des articles a été réalisée sur la base de critères bien définis au cours de la phase d'éligibilité (Annexe 1). Les critères d'inclusion comprennent : 1) les mots clés utilisés pour la recherche, croisés deux à deux, ce qui constitue la meilleure démarche (Dumez, 2011). Les duos de mots clés ainsi retenus ont été : mindfulness-work, mindfulness-workplace, mindfulness-organization, mindfulness-business, mindfulnessmarketing au moyen des opérateurs booléens OR et AND (certains mots clés ont été utilisé en français mais n'ont donné aucun résultat supplémentaire. Les articles retenus sont tous issus de la littérature anglo-saxonne) ; 2) les filtres sélectionnés : publications révisées par les pairs, publications comprises entre 2006-2017. Enfin, les critères d'exclusion concernent les publications n'ayant pas fait l'objet d'un classement CNRS, FNEGE ou sortant du cadre de la recherche. Nous avons ensuite sélectionné les contributions en examinant le classement des revues, la typologie des articles, la démarche de recherche, le secteur (entreprises privées : industrie, commerce, ou public : administration, santé...), ainsi que la population observée ou visée (employés, managers).

2.2 La méthode de traitement et d'analyse des données textuelles 
A l'issue de cette première sélection des articles, nous avons éliminé les articles qui ne contenaient pas au moins une fois le mot « mindfulness » ou « awareness » ou « mindful » dans leurs résultats. Nous avons ensuite élaboré un corpus avec les principales contributions des articles restants. Ce corpus est constitué de petits résumés de quelques lignes que nous avons rédigés pour chaque contribution d'article. Les résultats ont été trouvé ou repris en partie dans la discussion ou la conclusion de l'article. Afin d'obtenir une visualisation graphique du corpus, nous nous sommes alors posés la question du choix du logiciel de traitement de données et avons choisi TROPES (comme cela est expliqué dans le paragraphe suivant). Afin de faciliter l'interprétation, les fichiers ont ensuite été exportés dans un format GEXF adapté pour le logiciel graphique GEPHI. Tous les corpus et les fichiers GEXF ont été sauvegardés, classés et archivés dans un dossier.

\subsubsection{La posture épistémologique et le choix du logiciel}

Avant de choisir le logiciel de traitement des données, nous nous sommes posés des questions d'ordre épistémologique (Voynnet Fourboul, 2012). Dumez (2011, p. 57-58) défend l'idée que le chercheur doit inventer son approche en fonction du problème scientifique qu'il s'est construit. Dans notre démarche, de type interprétativiste, la recherche tente de comprendre et d'interpréter le sens global des contenus des corpus. L'analyse de données textuelles automatisée recouvre deux types d'approches : les analyses linguistiques et les analyses de contenu (Helme-Guizon et Gavard-Perret, 2004). TROPES se situe plutôt dans la famille analyse de contenu (Voynnet Fourboul, 2012). C'est un logiciel d'analyse de contenus textuels développé par le Groupe de Recherche sur la Parole (GRP) de l'université de Psychologie Paris VIII (Molette, 2009). Le principe du logiciel repose sur le regroupement des mots en catégories permettant de réduire le nombre de variables à classer ce qui permet d'explorer un texte et d'en dégager différents niveaux de sens et de valeurs (Ghiglione et al., 1998). Dans TROPES, le texte est découpé en unités de sens associés à des indices statistiques, les unités sont alors classées dans des catégories sémantiques et thématiques et font apparaître des associations (Helme-Guizon et GavardPerret, 2004 p. 76).

\subsubsection{La présentation de l'extension graphique GEPHI}

GEPHI est un logiciel d'extension graphique de licence libre compatible avec TROPES. Grâce à l'exportation des résultats de TROPES au format GEXF, les données peuvent être traitées par GEPHI et permettent de spatialiser les relations entre les variables (Molette, 2017). L'avantage est que certaines informations peuvent apparaître dans GEPHI alors 
qu'elles peuvent très bien ne pas l'être dans TROPES. Lorsque les données sont exportées au format GEXF, trois fichiers sont générés de manière automatique et peuvent ainsi être traités dans GEPHI : 1) la matrice des relations, 2) les cooccurrences entre les différents éléments du scénario (adapté pour les analyses d'entretiens), 3) la matrice des divers documents du scénario. La matrice des relations intéresse particulièrement notre étude, elle vise à mettre en lumière les principaux effets de la pleine conscience et les variables majeures étant en relation.

\section{Les principaux résultats}

\subsection{L'échantillon pour la revue systématique}

Le Tableau 1 suivant présente les différentes étapes de la construction de l'échantillon du 01 Novembre 2017 au 31 Janvier 2018. L'échantillon de présélection est composé de 136 articles sur la pleine conscience au travail et est ensuite fragmenté retenant 82 articles ayant fait l'objet de publications classées CNRS, FNEGE et s'intégrant aux objectifs de la recherche.

Tableau 1 : Etapes pour la construction de l'échantillon de la revue systématique

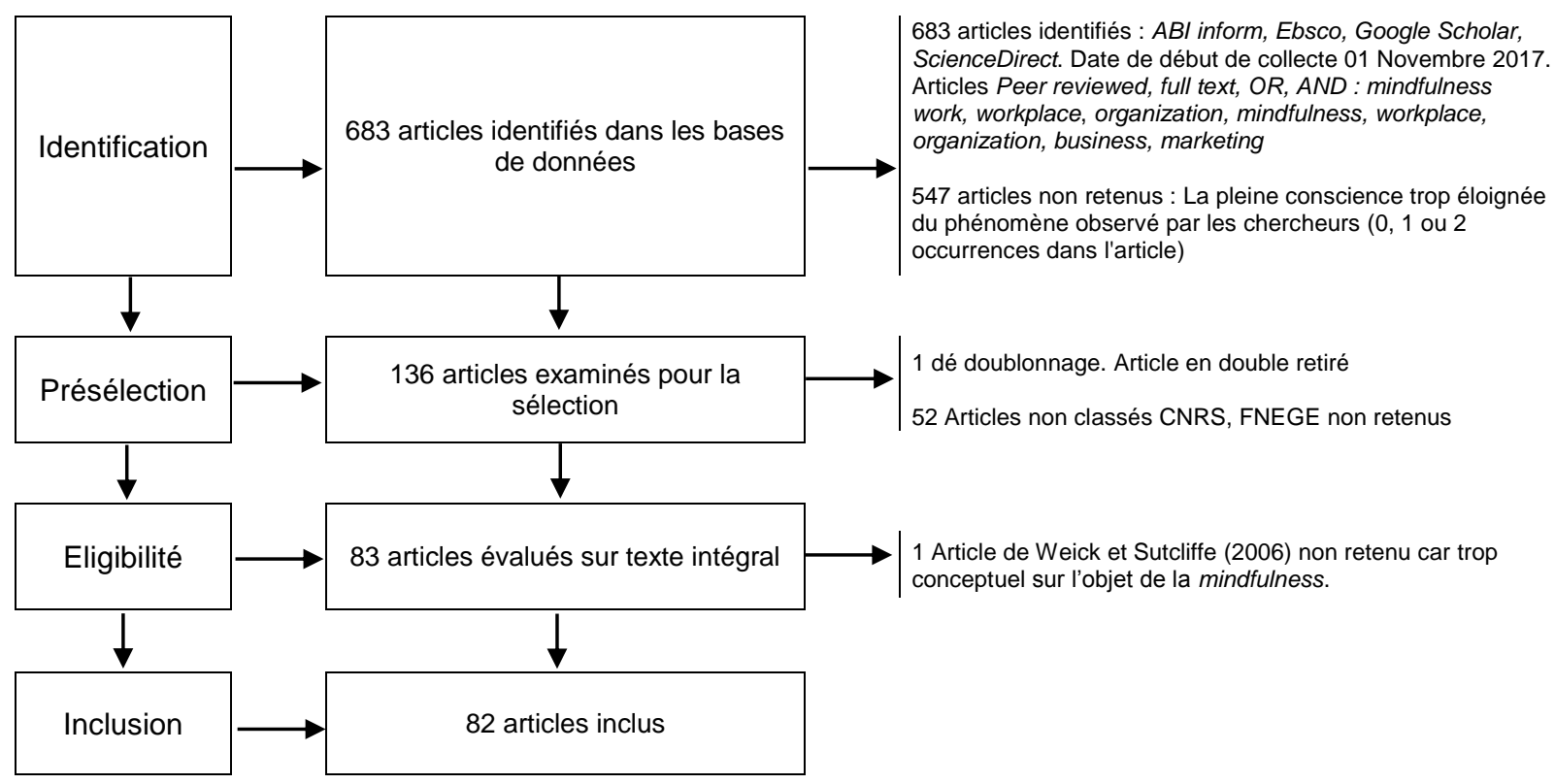

\subsection{La typologie des articles}

Les 82 publications se répartissent sur 33 revues différentes (Tableau 2). Entre 2006 et 2018, 14 articles sont parus dans Journal of Business Ethics ( $\left.1^{\text {er}}\right)$ et 12 dans Journal of Management Development ( $2^{\text {ème }}$ ) soit respectivement $17 \%$ et $14 \%$ de la totalité des articles. Ces deux revues représentent à elles seules $31 \%$, soit près d'un tiers de la totalité des 
articles publiés. Nous trouvons les notions d'éthique des affaires et de développement managérial dans les noms des 2 premières revues qui se rapportent au sujet de la pleine conscience au travail.

Tableau 2 : classement des revues selon le nombre de publications de 2006 à 2018

\begin{tabular}{|c|c|c|c|}
\hline Revues & CNRS & FNEGE & Nombre d'articles \\
\hline Journal of Business Ethics & 2 & 2 & 14 \\
\hline Journal of Management Development & 4 & 4 & 12 \\
\hline Occupational Health & & 3 & 6 \\
\hline Career Development International & & 4 & 4 \\
\hline Academy of Management Learning \& Education & 3 & 2 & 3 \\
\hline Journal of Management & 1 & 1 & 3 \\
\hline Journal of Managerial Psychology & 4 & 3 & 3 \\
\hline Journal of Occupational \& Organizational Psychology & 2 & 2 & 3 \\
\hline Personnel Review & 3 & 3 & 3 \\
\hline Journal of Management Inquiry & 3 & 2 & 3 \\
\hline human relations & 2 & 1 & 2 \\
\hline Journal of Business Research & 2 & 2 & 2 \\
\hline Journal of Happiness Studies & 4 & & 2 \\
\hline Journal of Vocational Behavior & 2 & 2 & 2 \\
\hline Management Decision & 4 & 3 & 2 \\
\hline Academy of Management Journal & 1 & 1 & 1 \\
\hline BMC Health Services Research & 3 & 3 & 1 \\
\hline Ecological Economics & 1 & & 1 \\
\hline European Business Review & 3 & 3 & 1 \\
\hline European Journal of Information Systems & 1 & 1 & 1 \\
\hline Group and Organization Management & 3 & 2 & 1 \\
\hline Information Technology \& People & 3 & 3 & 1 \\
\hline International Journal of Human Resource Management & 3 & 3 & 1 \\
\hline Journal of Applied Psychology & 1 & 1 & 1 \\
\hline Journal of Brand Management & 4 & 4 & 1 \\
\hline Journal of Organizational Behavior & 2 & 1 & 1 \\
\hline Journal of Organizational Change Management & 3 & 3 & 1 \\
\hline Journal of the Association for Information Systems & 2 & 2 & 1 \\
\hline MIT Sloan Management Review & & 2 & 1 \\
\hline Research in Organizational Behavior & 2 & 2 & 1 \\
\hline The Journal of Applied Behavioral Science & 3 & 3 & 1 \\
\hline The Learning Organization & 4 & 4 & 1 \\
\hline Total Quality Management Journal & 4 & & 1 \\
\hline
\end{tabular}

Parmi les 82 articles classés, nous avons détecté 3 types d'articles comme nous pouvons observer sur la Figure 3.

Figure 3 : Le type d'article 


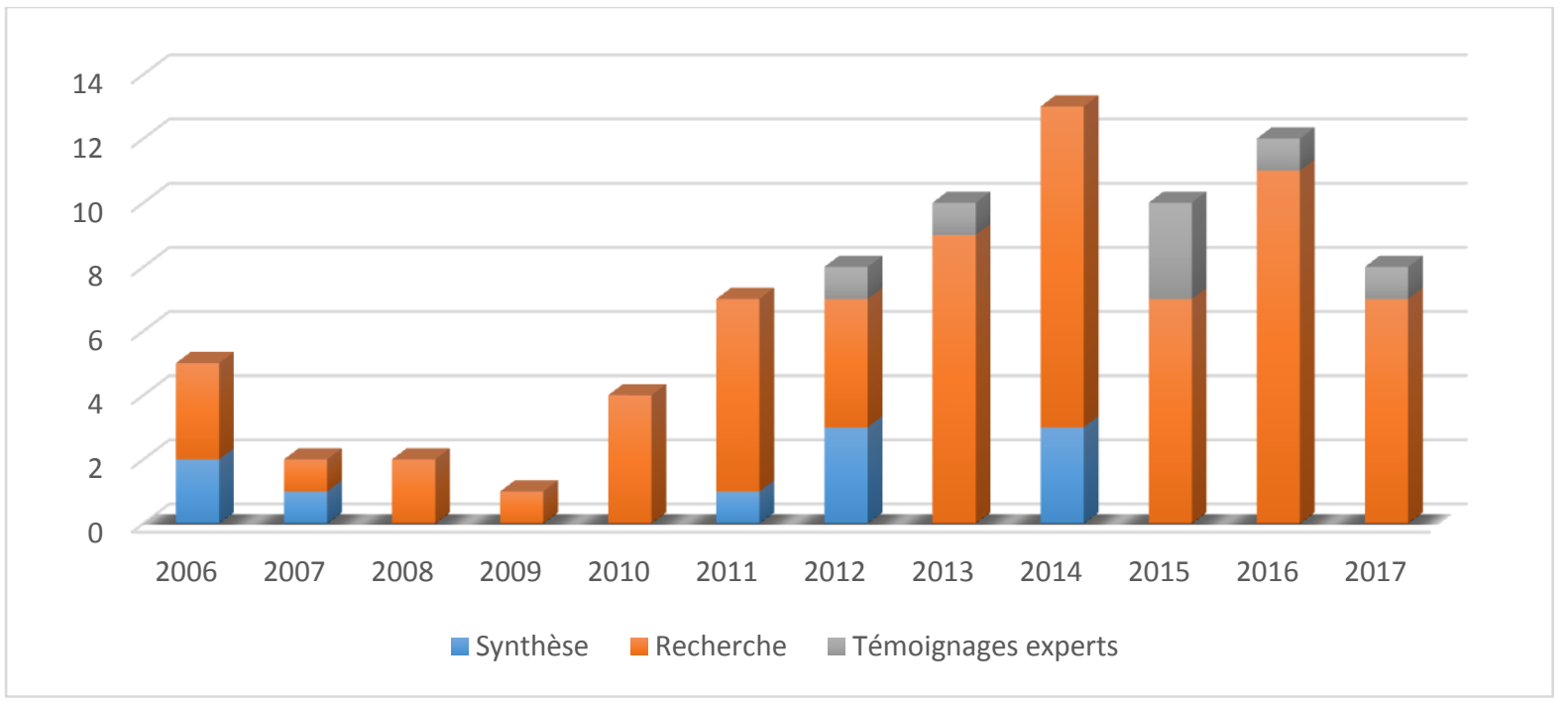

Les publications académiques relatives à la pleine conscience sont: 1) des articles de recherche (79\%) en constante progression depuis 2010 . 2) des articles de synthèse (12\%). 3 ) des témoignages d'experts (9\%). Les témoignages d'experts sont tous issus de la revue Occupational Health. Les personnes interrogées disposent souvent d'une expertise reconnue dans le domaine privé sur le sujet. Les informations recueillies sont souvent issues de l'expérience ou de l'observation du terrain professionnel par des experts du domaine.

\subsection{Les démarches de recherche}

Parmi les articles de recherche, quatre types de démarches de recherche scientifiques ont été identifiées dans la littérature sur la pleine conscience : 1) la revue de littérature (45\%). 2) la démarche quantitative (39\%) en progression constante depuis 2008 , 3) la démarche qualitative $(12 \%), 4)$ la démarche mixte $(4 \%)$. Ce résultat est présenté dans la Figure 4 suivante :

Figure 4 : Les démarches de recherche

- Revue de littérature - Démarche quantitative - Démarche qualitative - Démarche mixte

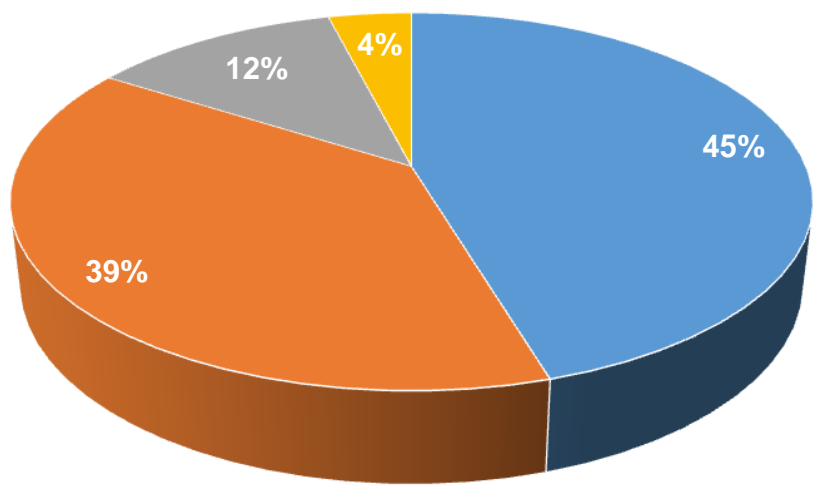




\subsection{Les secteurs d'activité}

Les secteurs d'activité, représentés dans la Figure 5, se répartissent ainsi : 1) secteur privé (56\% des articles), 2) le secteur public (administration, santé, 13\%) et, 3) le tout secteur confondu (pas de précision particulière sur le secteur visé, $31 \%$ ).

Figure 5 : Les secteurs d'activité

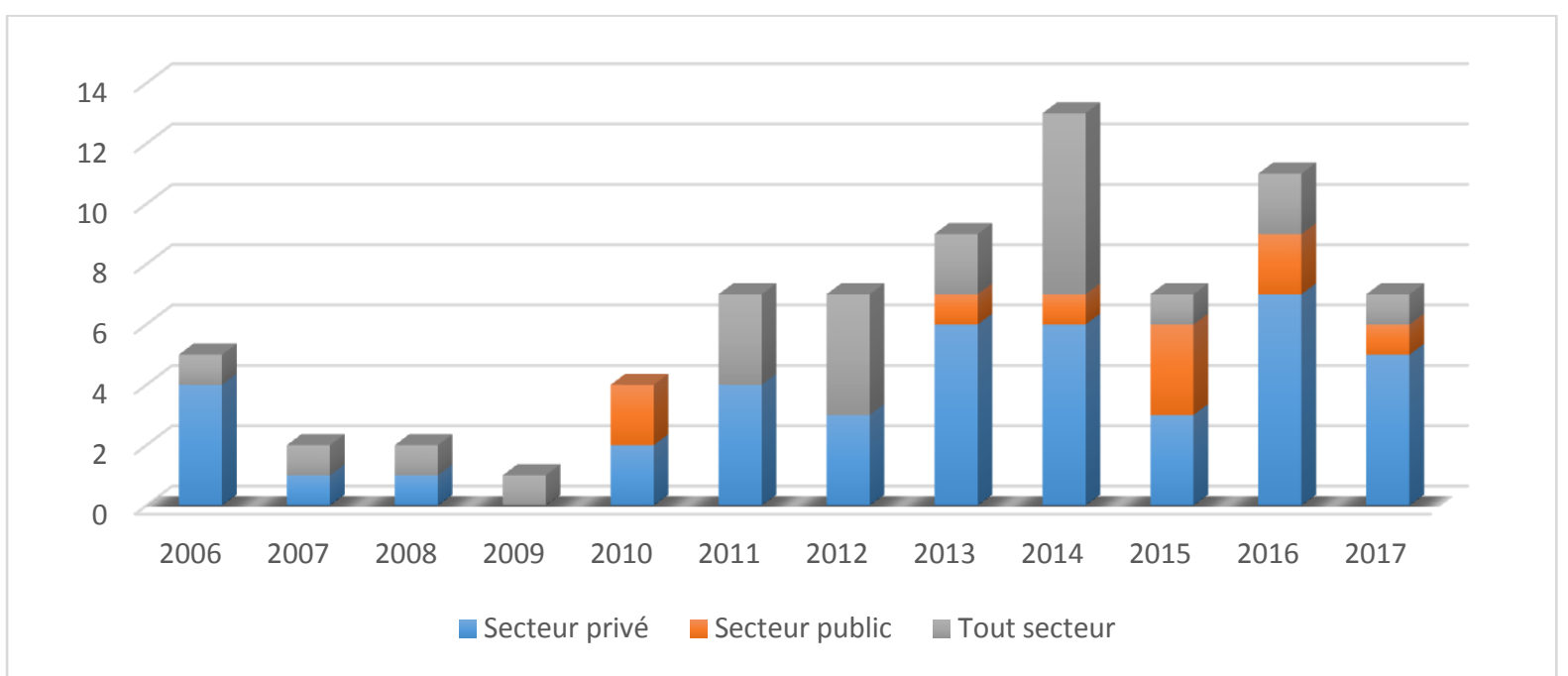

L'intérêt des études sur la pleine conscience en milieu professionnel dans tous les secteurs est certain mais c'est surtout dans le secteur privé que l'intérêt est le plus flagrant avec plus de la moitié des articles qui s'y consacrent. La tendance est également à la hausse depuis 2008.

\subsection{Les populations observées et visées}

Les populations sur lesquelles portent les études sont présentés dans la Figure 6 suivante. Ce sont : des employés (35\% des articles), des managers (32\%) et tout type personnel (pas de spécificité, 33\%).

Figure 6 : Les populations observées et visées 


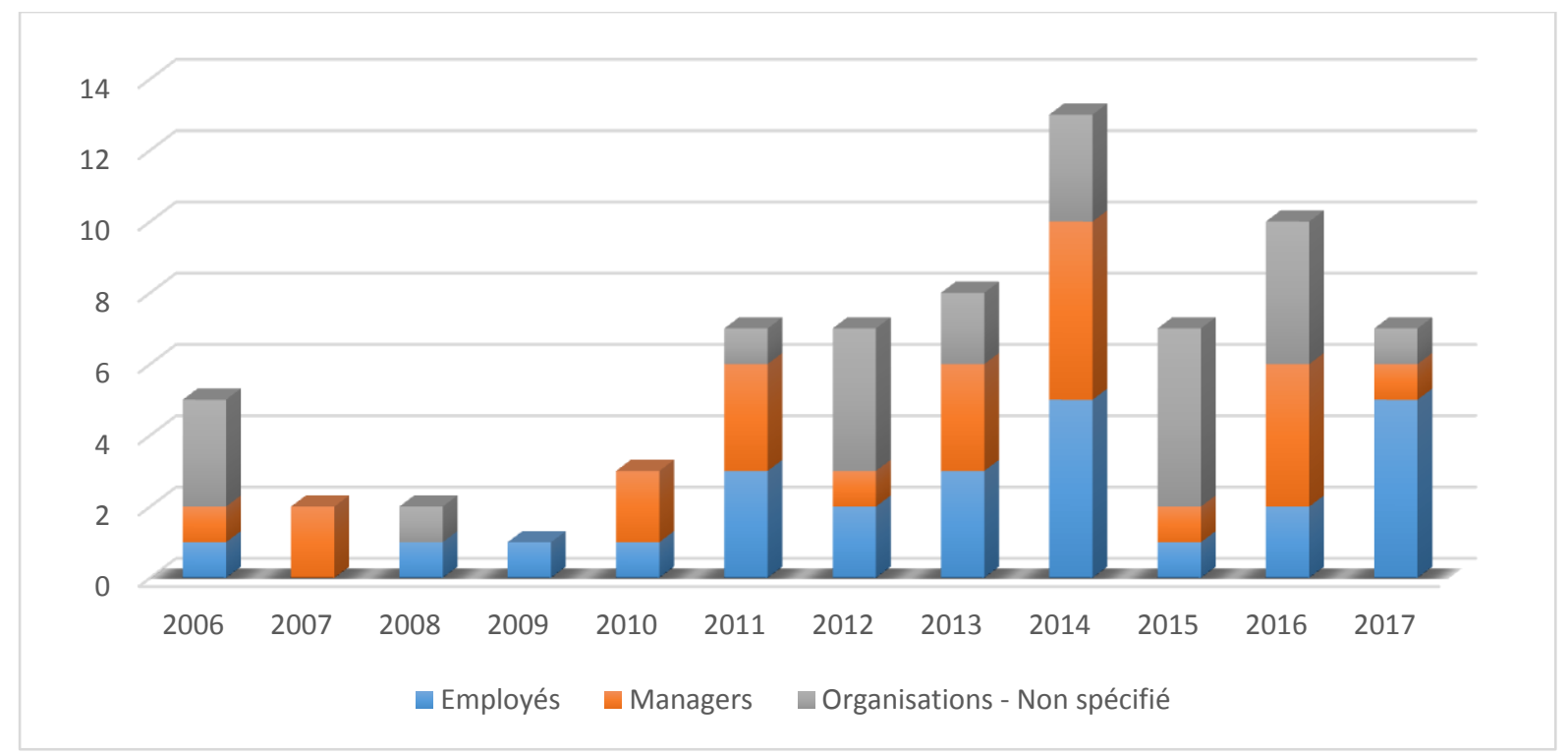

Les études intéressent tous les niveaux de l'organisation. En 2016 elles portaient surtout les managers alors que la tendance s'inverse de manière franche au profit des employés sur 2017.

\subsection{Les principales contributions des articles}

A ce stade, nous avons éliminé 28 articles qui ne contenaient pas le mot " mindfulness », « awareness » ou « mindful » dans les résultats et retenons les résultats de 54 articles au total. La visualisation graphique du réseau sémantique issu du corpus est présentée Figure 7. Nous proposons ensuite une analyse à quatre cadrans sur un plan orthogonal, en partant des nœuds de la périphérie vers le centre. La pleine conscience représente le centre. GEFI met automatiquement en relief les différents réseaux sémantiques concernés avec les nœuds et les relations en survolant le graphique avec souris de l'ordinateur. Nous obtenons ainsi les Figures 8, 9, 10 et 11 . Nous les interprétons en nous appuyant sur les principales références et principaux apports en exerçant des allers retours successifs avec la littérature.

Figure 7 : Le réseau de relations sémantiques de la pleine conscience au travail 


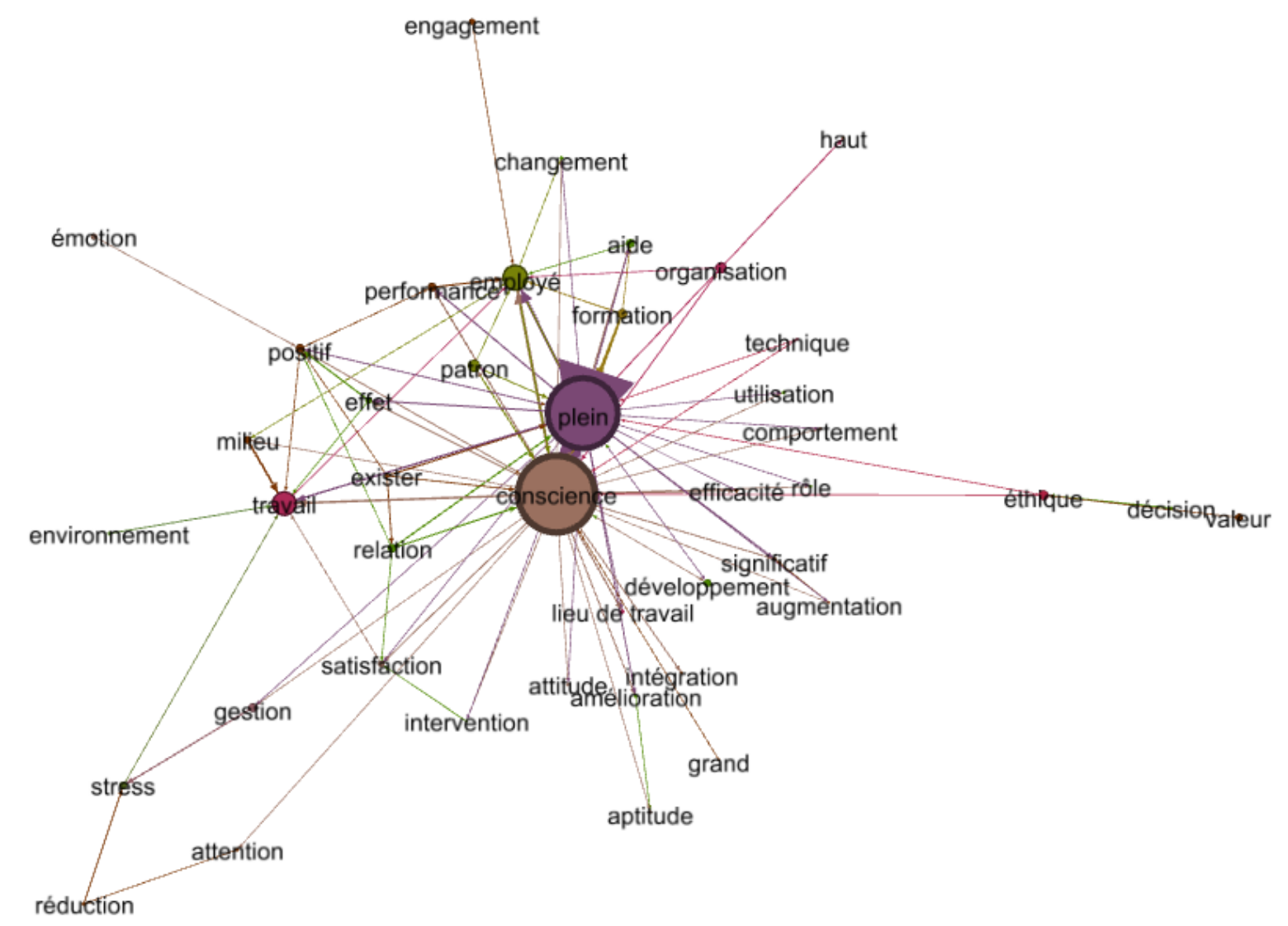

3.6.1 La pleine conscience, l'environnement de travail, le stress et la satisfaction au travail

La Figure 8 représente les relations entre la pleine conscience et l'environnement de travail. Elle fait apparaître 6 nœuds majeurs en lien: le stress, la satisfaction, le travail (l'environnement de travail), le positif, les employés, la pleine conscience. Les réactions affectives et le stress chez les employés sont principalement liés aux évènements ayant cours en milieu professionnel (Hülsheger et al., 2013 p. 312). Les causes du stress chez les employés dans les organisations sont nombreuses : environnements de travail changeants (Cacioppe, 2017 ; Charoensukmongkol, 2017), conflits de rôle (Valentine et al., 2010), surcharge d'informations (Bashford, 2012). La pleine conscience favorise le bien-être au travail (Marques et al., 2012). La formation à la méditation de pleine conscience en entreprise a un effet positif sur le bien-être perçu. Elle favorise l'attention et à la concentration ainsi qu'à un indicateur physiologique de réduction du stress (Baccarani et al., 2013). La pleine conscience et les techniques simples de respiration permettent aux employés de mieux gérer leur stress au moment opportun (Courtney, 2015). Elle améliore le bien-être de l'individu en diminuant l'anxiété et les ruminations mentales sur le passé et l'avenir. Elle permet de mieux gérer les réactions émotionnelles et développe la satisfaction 
au travail (Hülsheger et al., 2013 p. 313). Le mieux-être ainsi obtenu réduit l'intention de quitter l'entreprise ce qui favorise la réussite individuelle et collective (Zivnuska et al., 2016).

Figure 8: Réseau de relations entre la pleine conscience, l'environnement de travail, le stress et la satisfaction au travail

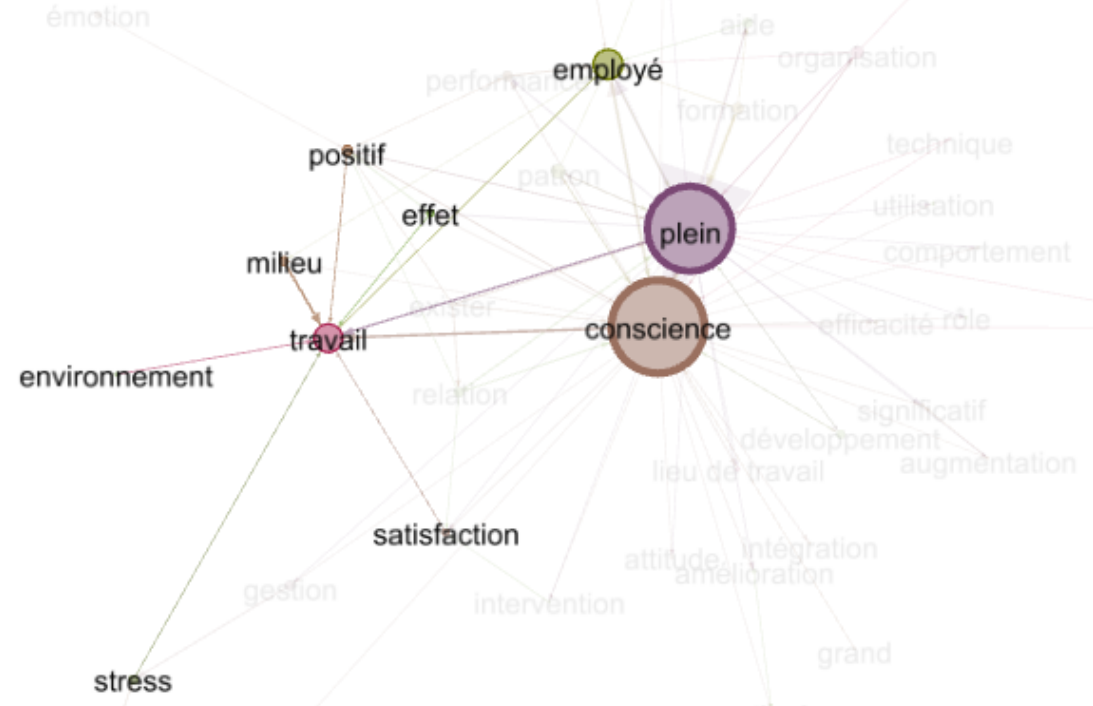

3.6.2 La pleine conscience, la gestion des émotions et la performance

Six nœuds majeurs en lien ressortent sur la Figure 9 : l'émotion, le positif, la relation, le travail, la performance, la pleine conscience. Les émotions au travail représentent un double intérêt pour les chercheurs et les praticiens. D'une part parce que les émotions et leur gestion jouent un rôle fondamental dans la communication et les relations interpersonnelles et d'autre parce que la gestion des émotions est un facteur d'épuisement professionnel. Tout d'abord, dans les relations, les émotions se transmettent. Le travail émotionnel des employés est devenu ainsi important afin que les émotions négatives soient contenues. L'environnement de travail confronte les employés à de nombreuses demandes. Les comportements autorégulés de contrôle de soi pour répondre à ces demandes puisent dans les ressources cognitives et émotionnelles. La pleine conscience, avec l'attention sur soi, 
permet une régulation autonome (désautomatisée) qui préserve l'énergie et la vitalité chez l'individu (Hülsheger et al., 2013 p. 312).

Figure 9 : Réseau de relations entre la pleine conscience et les émotions au travail

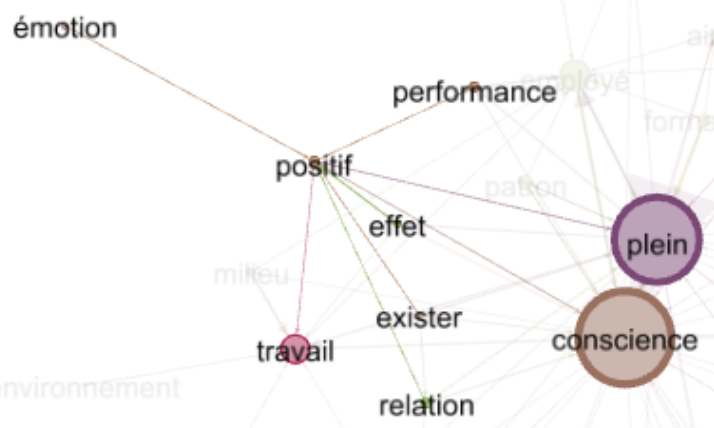

La pleine conscience permet une observation des évènements avec plus d'objectivité, sans y attacher d'opinion ou d'affect. Elle diminue ainsi l'épuisement émotionnel, et renforce les émotions positives (Avey et al., 2008 ; Andrews et al., 2014). Elle améliore l'écoute et la clarification des situations (Ucok, 2006 ; Gärtner, 2011). La pleine conscience favorise ainsi les relations interpersonnelles et la communication. La performance en lien avec les émotions a trait aux comportements relationnels et à la communication : une performance interpersonnelle. La pleine conscience permet de développer une écoute plus attentive des uns avec les autres. Ce qui permet de réduire les malentendus et la confusion, le temps et l'énergie gaspillés à réparer des erreurs (Ucok, 2006). De ce fait, la gestion des émotions et l'attention ainsi améliorées par la pleine conscience ont un effet négatif sur les comportements contre-productifs (déviance) et sur le risque d'erreurs (Good et al., 2016 p. 123).

3.6.3 La pleine conscience, l'engagement et la performance des employés 
Sur la Figure 10, nous voyons apparaitre sept nœuds principaux en lien : l'engagement, la performance, les employés, l'organisation, le travail, la formation, la pleine conscience. La pleine conscience par l'attention sur le moment présent implique plus d'engagement au travail et permet également plus d'authenticité par plus de conscience et de régulation de soi (Leroy et al., 2013). Les organisations peuvent retirer des avantages considérables de la formation de leurs employés à la pleine conscience en milieu de travail car elle permet l'acquisition de ressources qui profitent à l'employé mais aussi à l'organisation par la réussite professionnelle de ses employés grâce à un engagement accru (Zivnuska et al., 2016).

Figure 10 : Réseau de relations entre la pleine conscience, l'engagement et la performance des employés

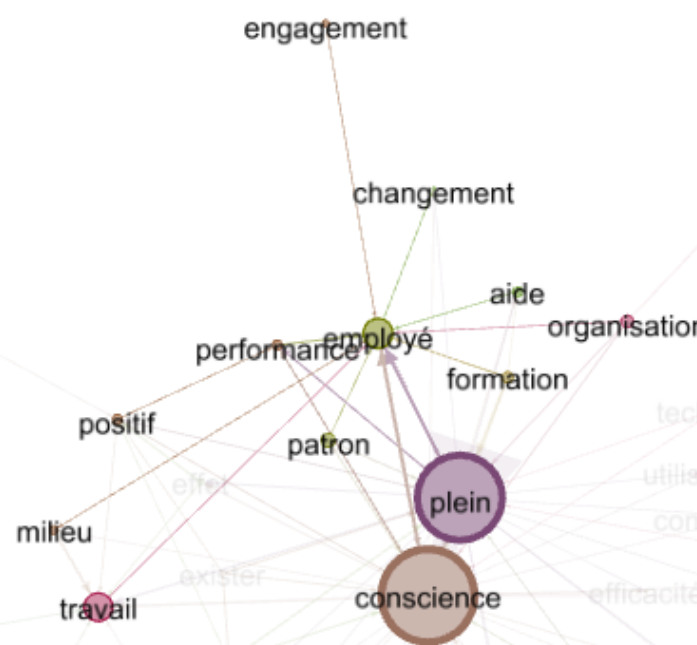

Concernant la performance en lien avec l'engagement, les résultats des articles mettent en avant trois types de performance qui semblent particulièrement impactés par la pleine conscience : la performance de tâche, la performance extra rôle et la performance créative. 1) La performance de tâche est souvent mesurée par le nombre de tâches à accomplir ou la diminution des erreurs d'inattention. La pleine conscience améliore la performance de tâche car elle développe la vigilance et la largeur attentionnelle. Elle permet aux individus d'éviter les erreurs qui se produisent quand l'attention se détourne du moment présent (Dane et 
Brummel, 2013 p. 109). Elle permet également de faire face à des situations parfois pénibles et génère plus de vitalité (Dane, 2011 p. 1004) ce qui peut avoir un effet dans la rapidité d'exécution de la tâche. 2) La performance extra rôle relève des comportements non prescrits, informels de l'employé comme la prise de certaines responsabilités. La pleine conscience génère plus de motivation intrinsèque et d'autonomie. L'attention sur le moment présent enrichit la qualité de l'expérience personnelle ce qui motive l'individu et le rend plus autonome dans la prise de décision de s'engager dans ces activités (Kroon et al., 2017 p. 286). 3) La performance créative. La pleine conscience en entreprise permet la créativité en offrant un plus grand espace d'innovation. Elle permet de développer différents styles de pensée créatives, de résolutions de problèmes, elle attise la curiosité (Hall, 2013 p. 16). L'engagement de l'individu dans le moment présent et l'ouverture sur le champ des possibles permettent de faire émerger la créativité pour soi et pour le groupe au travers du dialogue. La co-création peut ainsi mener à des pratiques commerciales plus durables, plus novatrices et profiter à l'ensemble de l'entreprise (Rill, 2016 p. 1144).

\subsubsection{La pleine conscience, les valeurs et l'éthique en entreprise}

Trois nœuds importants en lien sur la Figure 11 font surface: la valeur (les valeurs), l'éthique, la pleine conscience. L'éthique concerne $30 \%$ des études sur la pleine conscience chez les managers. L'éthique régit les principes moraux qui guident l'individu et la pleine conscience permet de développer la prise de décision éthique. L'éthique et la prise de décision éthique en entreprise dépendent de plusieurs facteurs :1) de codes de conduite personnels, 2) du climat éthique, 3) de la culture et de la formation à l'éthique, 4) des sanctions relatives à l'éthique, 5) de la taille de l'entreprise (Craft, 2013 p. 222). L'éthique d'un point de vue bouddhiste concerne « le respect de la vie et de la nature en général en chaque action » (Marques, 2012 p. 33). Elle est une des valeurs fondatrice de la culture bouddhiste avec la compassion, la bienveillance, l'action juste, les justes moyens d'existence, la non lutte, la collaboration et la charité (Ibid. p. 34). La pleine conscience est ainsi à l'origine de davantage de respect des règles morales (Verhezen, 2010). D'un point de vue managérial, le superviseur éthique obtient plus d'effort et d'aide du collaborateur quand il existe un haut niveau de pleine conscience et de sensibilité morale chez les collaborateurs (Eisenbeiss et Van Knippenberg, 2015). A l'éthique est souvent associée l'authenticité (Verhezen, 2010; Baron, 2014; Ketola, 2012). Ainsi, des dialogues authentiques et une formation à la prise de décision éthique en pleine conscience, peuvent susciter la confiance dans le respect des règles (Verhezen, 2010). Les managers qui parviennent à la pleine conscience sont également plus flexibles dans leurs comportements et l'utilisation du pouvoir (Karssiens et al., 2014). 
Figure 11 : La pleine conscience, les valeurs et l'éthique en entreprise

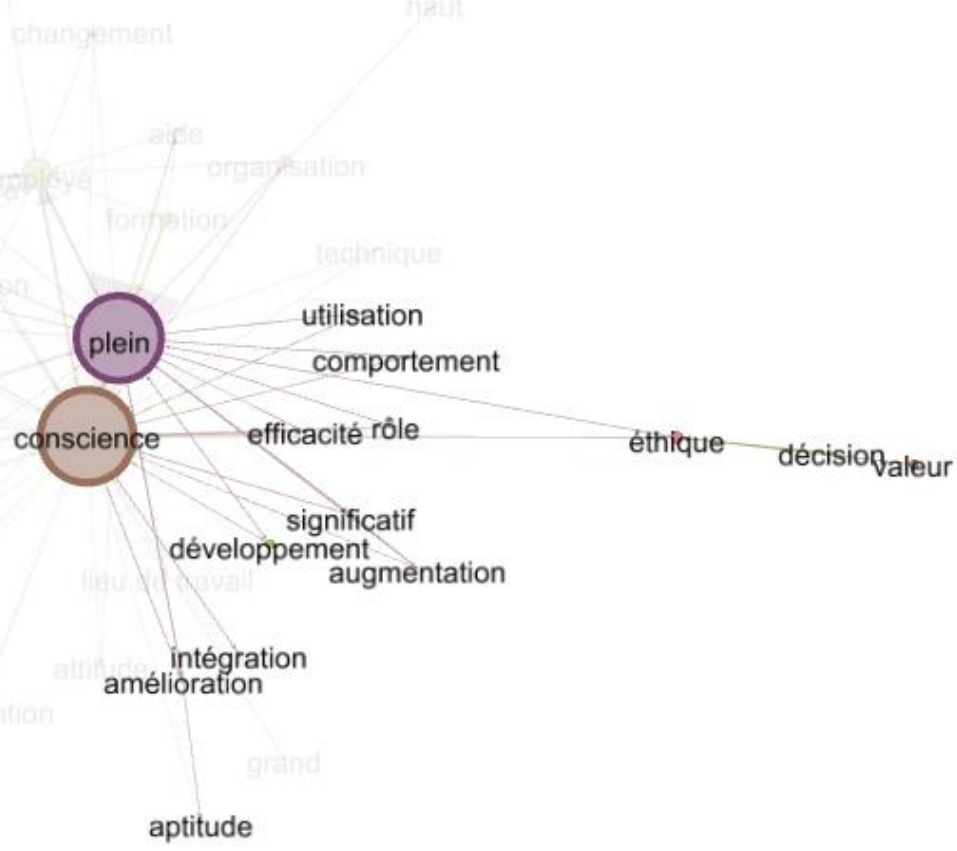

\section{Discussion et implications managériales}

Notre étude avait pour objectif d'analyser ce que la littérature scientifique a produit sur la pleine conscience au travail ces dix dernières années. L'intérêt pour la pleine conscience en milieu professionnel est en plein développement depuis 2010. Les recherches se veulent plus empiriques sur les bienfaits et les apports de ce phénomène en entreprise. Ce dernier touche les managers et les employés. L'analyse de contenu du corpus a permis de mettre en relief les variables majeures en lien avec la pleine conscience ce qui constitue l'intérêt principal de notre recherche. Nos résultats montrent que l'entreprise n'est pas seulement le lieu de la performance, mais aussi l'endroit où s'expriment les talents et où l'on vient pour s'épanouir, échanger. A un niveau plus macro, la responsabilité sociale des entreprises représente donc un enjeu fondamental. A ce titre, la question de l'adoption de la pleine conscience en entreprise se pose : la pleine conscience ne joue-t-elle pas un rôle majeur ? Agir en pleine conscience, n'est-il pas devenu une obligation sur le plan moral pour les managers ? Notre travail témoigne qu'au-delà de cette dimension morale, il existe un intérêt à développer la connaissance de la pleine conscience et de son mécanisme car celle-ci a 
des effets positifs sur les individus. La pleine conscience ne s'inscrit-elle pas alors dans un processus de développement durable des Ressources Humaines? Notre travail met en lumière l'importance de la pleine conscience en entreprise car elle est associée à de nombreuses qualités psychosociales. Elle peut servir à élaborer des outils de gestion notamment en termes de recrutement et de formation (Good et al., 2016 p. 134). La formation à la pleine conscience en entreprise peut alors démontrer ses avantages sur le développement durable des Ressources Humaines. Cependant, certaines versions de programmes de formation à la pleine conscience sont tronquées et mal adaptées par manque de connaissances (Good et al., 2016 p. 134). Comme le soulignent Dane et Brummel (2013), les qualités développées par la formation à la pleine conscience en entreprise dépendent de dispositions cognitives individuelles. II parait donc nécessaire de prendre en compte ces différences individuelles dans les programmes de formation à la pleine conscience en envisageant des designs de formation adaptés. De plus, l'intégration de la pleine conscience en entreprise reste compliquée de par ses origines. Une intégration par les valeurs est moins problématique (Marques, 2012). Afin de faciliter l'intégration de la pleine conscience en entreprise nous pouvons suggérer un tableau de bord managérial fondé sur les valeurs de la pleine conscience. Ce tableau de bord, pourrait être constitué d'une liste d'items en lien avec les valeurs fondatrices de la pleine conscience issues de la philosophie bouddhiste (éthique individuelle, compassion, bienveillance, charité). II permettrait une évaluation de la part du superviseur de ces valeurs. Ce tableau de bord pourrait être utilisé lors des recrutements, ou utilisés lors des suivis d'évaluation, d'actions de formation ou de supervision managériale. Au recrutement ce tableau permet une mesure des valeurs personnelles des individus en lien avec les valeurs de la pleine conscience. En suivi d'évaluation ou supervision, les ajustements se font sous la forme d'actions de formation et de travail sur les valeurs. Toujours en termes d'implications managériales, nous pouvons proposer un label de l'entreprise mindfulness. Ce label pourrait s'obtenir selon des critères liés aux valeurs de la pleine conscience en entreprise, il serait un élément différenciant, pouvant renforcer l'attractivité de l'entreprise.

\section{Les limites et voies futures de recherche}

Malgré notre approche systématique et rigoureuse basée sur l'analyse de contenu, notre recherche présente certaines limites. En effet, afin d'intégrer les nombreuses perspectives et significations données à la pleine conscience nous nous sommes focalisés sur les fondements communs en négligeant les spécificités contextuelles ce qui constitue une première limite et aussi une piste de recherche de ce travail. Une deuxième limite concerne le risque d'ethnocentrisme de notre étude. Comme l'expliquent Leca et Ple (2013, p.37), 
"Les textes scientifiques renferment des stratégies discursives visant à convaincre le lecteur, autant qu'à présenter des faits. Geertz ne critique pas ceci mais appelle simplement les chercheurs à en prendre conscience et en conséquence à admettre la fragilité de tout texte scientifique ». "Ce qui est central est l'humilité doit faire preuve le chercheur ». D'un point de vue ethnique ou anthropologique, la pleine conscience a traversé les civilisations, et les siècles sous divers courants philosophiques et religieux. Ces courants se sont emparés du phénomène et y ont donné des appellations, analogies au divin et au spirituel. Notre article a voulu apporter un éclairage sur ce phénomène universel à l'aide d'une approche scientifique. L'idée de cet article fût de considérer la pleine conscience hors de ses dimensions mystiques et ésotériques afin de comprendre que la pleine conscience est un phénomène naturel chez l'individu. Ce phénomène peut être bénéfique aux ressources humaines de l'entreprise, c'est ce que nous avons voulons mettre en évidence. Une troisième limite concerne les unités d'analyse constituées pour réaliser le corpus, elles respectent au maximum la neutralité et le sens voulu par les auteurs mais elles passent par notre formulation pour une meilleure lecture par le logiciel TROPES. Enfin, une dernière limite concerne l'engagement et les différentes catégories de performance: notre étude montre que la pleine conscience a des effets positifs sur l'engagement avec des conséquences positives sur la performance de tâche, la performance extra-rôle (de citoyenneté) et la performance créative. Comme le soulignent Dane et Brummel (2013), il est d'importance de mieux prendre en compte le dévouement dans le concept de l'engagement car tous les individus n'ont pas le même degré d'attachement envers leur employeur. Plusieurs pistes de recherche découlent de cette revue de la littérature. Ces pistes émergent directement des résultats de ce travail : de futures études pourraient porter sur des variables mises en avant par les articles examinés. Une première piste de recherche concerne la gestion de stress et la satisfaction au travail. Notre étude, en s'appuyant sur les résultats des chercheurs, met en relief la relation positive entre la pleine conscience et la satisfaction au travail avec la gestion de stress comme médiateur de la relation. Comme le montrent Zivnuska et al. (2017), et selon la théorie de l'autorégulation RFT (regulationfocused theory), les individus utilisent des stratégies différentes pour atteindre leurs objectifs professionnels. Certains sont motivés par l'idée de promotion (promotion-focused) et d'autres sont d'avantage motivés par l'évitement au risque et à la souffrance (preventionfocused). Dans leur étude, les auteurs démontrent que les individus motivés par l'évitement au risque sont plus sujets au stress. II semble donc intéressant de prendre en compte l'effet de ces deux variables (promotion et prevention) en tant que modérateurs dans le cadre d'études portant sur la pleine conscience, le stress et la satisfaction au travail. Une deuxième piste de recherche porte sur les valeurs, l'éthique et la prise de décision éthique. Notre travail de recherche met en lumière l'intérêt de la formation à la pleine conscience 
dans la prise de décision éthique, le respect des règles, l'authenticité. Néanmoins l'intégration de la pleine conscience en entreprise reste compliquée de par ses origines bouddhistes (Marques, 2010). Une intégration par les valeurs est moins problématique (Kernochan et al., 2007). L'éthique, l'acceptation, l'ouverture, associés à certains concepts bouddhistes revisités de non-lutte, de compassion, pourraient servir de point de départ pour développer la pleine conscience en entreprise (Marques, 2012). Il est donc intéressant de s'interroger sur la façon d'amener la formation à la pleine conscience dans la prise de décision éthique en entreprise. Une autre piste émerge de ce travail et concerne la prise de décision éthique chez les employés. Présente chez les managers, elle semble absente de notre revue de la littérature chez les employés.

\section{Conclusion}

Ce travail est basé sur une analyse minutieuse de 82 articles récents publiés dans des revues de sciences de gestion classées. Les résultats de l'analyse quantitative dressent un panorama des études sur la pleine conscience au travail et présentent une vision relativement exhaustive des articles parus sur la pleine conscience en milieu professionnel. Ces résultats sont ensuite analysés en profondeur et explicités avec les principales contributions des études correspondantes. L'analyse de contenu nous a permis d'identifier les variables clés reliées à la pleine conscience. Ceci constitue l'apport principal de notre travail. Un autre apport réside dans la démarche scientifique : la revue systématique de la littérature complétée par une analyse des principaux résultats qui permet d'avoir une vision plus précise des enjeux de la pleine conscience. Finalement, notre étude propose des voies concrètes de recherche. Les investigations suggérées permettront une meilleure compréhension du concept de pleine conscience dans des contextes de services dans un but aussi bien managérial qu'académique. A l'issue de cette synthèse réalisée selon une démarche scientifique rigoureuse, il apparaît que le champ de recherche sur la pleine conscience est en pleine construction. Toutefois, la multiplication des recherches récentes sur ce sujet témoigne aussi d'un fort engouement des chercheurs pour ce thème.

\section{Références}

Abord de Chatillon, E., Bachelard, O. (2006). Introduction générale au cahier spécial santé, sécurité au travail. Management \& Avenir, p. 121-127.

Akin, U., Akin, A., Ugur, E. (2016). Mediating Role of Mindfulness on the Associations of Friendship Quality and Subjective Vitality. Psychological Reports, p. 516-526. 
Allen, T.D., Kiburz, K.M. (2012). Trait mindfulness and work-family balance among. Journal of Vocational Behavior, p. 372-379.

Andrews, M.C., Kacmar, K.M., Kacmar, C. (2014). The mediational effect of regulatory focus on the relationships between mindfulness and job satisfaction. Career Development International, p. 494-507.

Avey, J.B., Wersing, T.S., Luthans, F. (2008). Can Positive Employees Help Positive. The Journal of Applied Behavioral Science, p. 48-70.

Baccarani, C., Mascherpa, V., Minozzo, M. (2013). Zen and well-being at the workplace. Total Quality Management Journal, p. 606-624.

Baron, L. (2016). Authentic leadership and mindfulness development through action learning. Journal of Managerial Psychology, p. 296 - 311.

Bashford, S. (2012). Staying calm: it's the thought that counts. Occupational Health, p. 21 23.

Bishop, S.R., Lau, M., Shapiro, S., Carlson, L., Anderson, N.D., Carmody, J., Segal, Z.V., Abbey, S., Speca, M., Velting, D., Devins, G. (2004). Mindfulness: A Proposed Operational Definition. Clinical Psychology: Science and Practice, p. 230-241.

Brendel, W., Hankerson, S., Byun, S., Cunningham, B. (2016). Cultivating leadership Dharma: Measuring the impact of regular mindfulness practice on creativity, resilience, tolerance for ambiguity, anxiety and stress. Journal of Management Development, p. 1056-1078.

Brown, K.W., Ryan, R.M. (2003). The benefits of being present: Mindfulness and its role in psychological well-being. Journal of Personality and Social Psychology, p. 822-848.

Brown, K.W., Ryan, R.M., Cresswell, J.D. (2007). Mindfulness: Theoretical foundations and evidence for its salutary effects. Psychological Inquiry, p. 211-237.

Cacioppe, R.L. (2017). Integral mindflow: A process of mindfulness-in-flow to enhance. The Learning Organization, p. 408-417.

Charoensukmongkol, P. (2017). Contributions of mindfulness during post-merger integration. Journal of Managerial Psychology, p. 104-118.

Courtney, J. (2015). Can improving "personal resilience" reduce absence due to stress? . Occupational Health, p. 10.

Craft, J.L. (2013). A Review of the Empirical Ethical Decision-Making Literature: 2004-2011. Journal of Business Ethics, p. 221-259.

Dane, E. (2011). Paying Attention to Mindfulness and Its Effects on Task Performance in the Workplace. Journal of Management, p. 997-1018.

Dane, E., Brummel, B.J. (2013). Examining workplace mindfulness and its relations to job performance and turnover intention. human relations, p. $105-128$.

Davids, T.W.R., Stede, W. (1959). Pali-English Dictionary. London: Luzac \& Company, Ltd., 813 pages.

De Bruyn, P.H. (2014). Le taoïsme, chemins de découverte. Paris: CNRS éditions, 282 pages.

Desreumaux, A. (2015). Théorie des organisations - 3e édition. Caen: EMS ; Management et Société, 312 pages. 
Dumez, H. (2011). Faire une revue de littérature : pourquoi et comment ? Le Libellio d' AEGIS, p. 15-27.

Durach, C.F., Kembro, J., Wieland, A. (2017). A new paradigm for systematic litterature reviews in supply chain management. Journal of Supply Chain Management, p. 6785.

Eisenbeiss, S.A., Van Knippenberg, D. (2014). On ethical leadership impact: The role of follower mindfulness and moral emotions. Journal of Organizational Behavior, p. 182-195.

Erisman, S.M., Roemer, L. (2012). A Preliminary Investigation of the Process of Mindfulness. Mindfulness, p. 30-43.

Feuvrier, M.P. (2014). Bonheur et travail, oxymore ou piste de management stratégique de l'entreprise ? Management \& Avenir, p. 164-182.

Françoise, D. (2016). Pleine-conscience et management : effet de la mindfulness sur la relation au travail et le style de leadership des managers. Thèse: 455 pages.

Freeman, L. (2007). Lettres sur la méditation : le christianisme face au silence. Paris: Albin Michel spiritualités, 240 pages.

Gärtner, C. (2011). Putting new wine into old bottles Mindfulness as a micro-foundation of dynamic capabilities. Management Decision, p. 253-269.

Ghiglione, R., Landre, A., Bromberg, M., Molette, P. (1998). L'Analyse automatique des contenus (Psycho Sup ed.). Paris: Dunod. 168 Pages.

Gintrac, A. (2011). Le stress au travail, un état des lieux. Management \& Avenir, p. 89-106.

Glomb, T.M., Duffy, M.K., Bono, J.E., Yang, T. (2012). Mindfulness at work. Research in Personnel and Human Resources Management : Emerald Publishing Limited, p. 115-157.

Good, D., Lyddy, C.J., Glomb, T.M., Bono, J.E., Brown, W., Duffy, K., Baer, M.K., Brewer, R., Lazar, S.W. (2016). Contemplating Mindfulness at Work: An Integrative Review. Journal of Management, p. 1-29.

Gregoire, S., Lachance, L., Richer, L. (2016). La présence attentive - Mindfulness. Québec: Presses de l'université du Québec. 268 Pages.

Grenier, C., Martin, V. (2013). Performance des organisations et bien-être des usagers : quels modes de pilotage et d'intervention? Management \& Avenir, p. 130-145.

Hall, L. (2013). Focusing on the good things. Occupational Health, p. 15-17.

Hayes, S. C., Luoma, J. B., Bond, F. W., Masuda, A., Lillis, J. (2006). Acceptance and Commitment Therapy: Model, processes and outcomes. Behaviour Research and Therapy, p. 1-31.

Helme-Guizon, A., Gavard-Perret, M.L. (2004). L'analyse automatisée de données textuelles en marketing : Comparaison de trois logiciels. Décisions Marketing, 36; Business Premium Collection, p. 75-90.

Hülsheger, U.R., Alberts, H.J.E.M., Feinholdt, A., Lang, W.B. (2013). Benefits of Mindfulness at Work: The Role of Mindfulness in Emotion Regulation, Emotional Exhaustion, and Job Satisfaction. Journal of Applied Psychology, p. 1-16. 
Kabat-Zinn, J. (2003). Mindfulness-Based Interventions in Context: Past, Present, and Future. Clinical Psychology: Science and practice, p. 144-156.

Kane, O. (2013). La communication entre présence et absence : Une relecture sémiotique de la "querelle des images" et de la mystique Soufie. Canadian Journal of Communication, p. 517-530.

Karssiens, A. E. A., Van Der Linden, C., Wilderom, C.P.M., Furtmueller, E. (2014). Embodied Mind Knowledge in Leadership Practice: Creating Space in Patterned Thoughts and Behaviors. Journal of Management Inquiry, p. 231-241.

Kernochan, R.A., McCormick, D.W., White, J.A. (2007). Spirituality and the Management Teacher. Journal of Management Inquiry, p. 61-75.

Ketola, T. (2012). Losing your self: managerial persona and shadow pressures killing responsible leadership. Journal of Management Development, p. 470-487.

Kroon, B., Van Woerkom, M., Menting, C. (2017). Mindfulness as substitute for transformational leadership. Journal of Managerial Psychology, p. 284-297.

Le Flanchec, A., Mullenbach-Servayre, A., Rojot, J. (2015). Favoriser la satisfaction au travail : les apports de l'enquête REPONSE 2011. Management \& Avenir, p. 37-55.

Langer, E.J., Moldoveanu, M. (2000). The construct of Mindfulness. Journal of social Issues, p. 1-9.

Leca, B., Ple, L. (2013). Une épistémologie à hauteur d'homme : l'anthropologie interprétative de Clifford Geertz et son apport potentiel à la recherche francophone en management. Management \& Avenir, p. 35-52.

Leroy, H., Anseel, F., Dimitrova, N.G., Sels, L. (2013). Mindfulness, authentic functioning, and work engagement: A growth modeling approach. Journal of Vocational Behavior, p. 238-247.

Ludwig, Q. (2006). Comprendre la kabbale : de Rabbi Siméon bar Yochaï à Madonna. Paris: Eyrolles, 201 pages.

Marques, J. (2010). Making Buddhism work @ work: the transformation of a religion into a seasoned ethical system. Journal of Management Development, p. 537-549.

Marques, J. (2012). Consciousness at Work: A Review of Some Important Values, Discussed from a Buddhist Perspective. Journal of Business Ethics, p. 27-40.

Marques, J., Dhiman, S.K., Biberman, J. (2012). Teaching the un-teachable: storytelling and meditation. Journal of Management Development, p. 196-217.

McElroy, M.W., Jorna, R.J., Van Engelen, J. (2006). Rethinking social capital theory: a knowledge management perspective. Journal of Knowledge Management, p. 124136.

Molette, P. (2009). De l'APD à Tropes : comment un outil d'analyse de contenu peut évoluer en logiciel de classification sémantique généraliste. Tarbes: Communication au colloque Psychologie et communication.Consulté le 15-08-2018, sur : https://www.tropes.fr/PierreMoletteCommunicationColloquePsychoTarbesJuin2009.pdf

Molette, P. (2017). Extensions Gephi Gexf de Tropes. Consulté sur Tropes le 15-08-2018: https://www.tropes.fr/UtiliserGephiavecTropesVF84.pdf

Pavie, X. (2010). La méditation philosophique. Paris: Eyrolles, 124 pages. 
Pirson, M.A., Langer, E.J., Bodner, T.E., Zilcha-Mano, S. (2012). The Development and Validation of the Langer Mindfulness Scale - Enabling a Socio-Cognitive Perspective of mindfulness in organizational contexts. SSRN Electronic Journal, p. 1-55.

Rill, B. (2016). Resonant co-creation as an approach to strategic innovation. Journal of Organizational Change Management, p. 1135-1152.

Ruedy, E.N., Schweitzer, M.E. (2010). In the Moment: The Effect of Mindfulness. Journal of Business Ethics, p. 73-87.

Seignour, A. (2011). Méthode d'analyse des discours : l'exemple de l'allocution d'un dirigeant d'entreprise publique. Revue française de Gestion, p. 29-45.

Shapiro, S.L., Carlson, L.E., Astin, J.A., Freedman, B. (2008). Mechanisms of Mindfulness. Journal of Clinical Psychology, p. 373-386.

Swierczek, F.W., Jousse, D. (2014). Adam Smith as Bodhisattva? A metta analysis of global leadership. Journal of Management Development, p. 786-796.

Tan, C.M. (2015). Just 6 Seconds of Mindfulness Can Make You More Effective. Harvard Business Review. Consulté le 14-02-2018 sur : https://hbr.org/2015/12/just-6seconds-of-mindfulness-can-make-you-more-effective. p.1-4

Thomas, D.C. (2006). Domain and Development of Cultural Intelligence: The Importance of Mindfulness. Group and Organization Management, p. 78-99.

Tranfield, D., Denyer, D., Smart, P. (2003). Towards a Methodology for Developing Evidence-Informed Management Knowledge by Means of Systematic Review. British Journal of Management, p. 207-222.

Ucok, O. (2006). Transparency, communication and mindfulness. Journal of Management Development, p. 1024-1028.

Valentine, S., Godkin, L., Varca, P.E. (2010). Role Conflict, Mindfulness, and Organizational Ethics in an Education-Based Healthcare Institution. Journal of Business Ethics, p. 455-469.

Varenne, J. (1990). Upanishads du yoga . Paris : Gallimard, 167 pages.

Verhezen, P. (2010). Giving Voice in a Culture of Silence. From a Culture of Compliance to a Culture of Integrity. Journal of Business Ethics, p. 763-775.

Voynnet Fourboul, C. (2012). Ce que "analyse de données qualitatives" veut dire. Revue internationale de psychosociologie, p. 71-88.

Walsh, R., Shapiro, S.L. (2006). The Meeting of Meditative Disciplines and Western Psychology. American Psychologist, p. 227-239.

Weick, K.E., Putnam, T. (2006). Organizing for Mindfulness Eastern Wisdom and Western Knowledge. Journal of Management Inquiry, p. 275-287.

Zivnuska, S., Kacmar, K. M., Ferguson, M., Carlson, D.S. (2016). Mindfulness at work: resource accumulation, well-being, and attitudes. Career Development International, p. 106-124.

Zivnuska, S., Kacmar, K. M., Valle, M. (2017). The mechanisms of regulatory focus: Mindfulness, leader-member exchange, and motivational outcomes. Career Development International, p. 37-49. 